\title{
Exploratory Analysis of Superposition Coding and Rate Splitting for Multibeam Satellite Systems
}

\author{
Màrius Caus*, Adriano Pastore*, Mònica Navarro*, Tomás Ramírez ${ }^{\dagger}$, Carlos Mosquera $^{\dagger}$, Nele Noels $\ddagger$ \\ Nader Alagha ${ }^{\S}$ and Ana I. Perez-Neira*ף \\ * Centre Tecnològic de Telecomunicacions de Catalunya (CTTC/CERCA), Castelldefels, Barcelona, Spain \\ $\dagger$ atlanTTic research center, University of Vigo, Galicia, Spain \\ $\ddagger$ DIGCOM research group, TELIN Department, Ghent University, Ghent, Belgium \\ ${ }^{\S}$ European Space Agency (ESA-ESTEC), Noordwijk, Netherlands \\ IDept. of Signal Theory and Communications, Universitat Politècnica de Catalunya (UPC), Barcelona, Spain
}

\begin{abstract}
The adoption of aggressive frequency reuse schemes along with interference management techniques has become the leading paradigm in satellite communications to increase the spectral efficiency. In general terms, one cannot rely on precoding techniques in the absence of channel phase information. Nevertheless, the availability of channel magnitude information, makes it possible to explore power-based separation of superimposed signals. In this paper, rate splitting (RS) ideas are exploited, whereby the separation of messages into private and public parts serves to improve the performance of successive cancellation decoding (SCD). Numerical results reveal that in some pertinent system scenarios, the proposed schemes achieve a larger rate region than that of orthogonal schemes that do not exploit the interference and other strategies that either do not allow beam cooperation or do not apply RS.
\end{abstract}

\section{INTRODUCTION}

The ever-growing demand for throughput has prompted research efforts to improve the spectral efficiency of communication systems. In the satellite context, a very promising approach consists in creating a multibeam radiation pattern, with a reduced distance between co-channel beams. This observation highlights that future satellite communications systems should rely on interference management techniques.

To mitigate co-channel interference, we can benefit from the analogy that can be drawn between the forward link of a multibeam satellite system and the multiple-input singleoutput broadcast channel (MISO-BC). This observation highlights that the interference can be pre-canceled at the transmit side by resorting to precoding techniques [1]-[3].

The lack of accurate channel state information at the transmitter (CSIT) induces multi-user interference, which becomes the main obstacle for implementing precoding techniques. Recent studies have shown that rate splitting (RS) combined with linear precoding techniques provides benefits in terms of spectral efficiency, when a perfect CSIT is not available [4].

This paper delves into RS and superposition coding (SC) ideas to increase the spectral efficiency gains of multibeam satellite systems, while significantly reducing the feedback overhead. That is, without using full CSIT, but only the amplitude of the channel. Under this constraint, two techniques that lie within the 2 user MISO-BC framework are investigated.
The first coding scheme that is investigated is referred to as SC with successive cancellation decoding (SC-SCD). It is well known that this scheme is able to achieve the capacity of degraded broadcast channels [5]. The MISO-BC, which is the theoretical framework of multibeam satellite communication systems, is not in general degraded and thus, SC-SCD is suboptimal for this class of channels. However, the use of SCSCD is endorsed by the possibility of applying low-complexity encoding strategies that do not require full CSIT.

The second technique is inspired by the interference enhancement $(\mathrm{ENH})$ scheme, which is proposed to increase the generalized degrees of freedom (GDoF) [6]. It must be mentioned that the architecture presented in [4] is sufficiently general to accommodate the ENH scheme. Unfortunately, the maximum achievable rates of ENH are only attainable if the channel phase information is known by the transmitter. To overcome this issue the non coherent rate splitting (NCRS) scheme is presented.

Numerical results for two users scenario reveal that the proposed schemes clearly outperform solutions based on frequency division multiplexing (FDM) and other strategies that either do not leverage on RS or do not allow full beam cooperation. The SC-SCD solution only gives satisfactory performance when one of the users experiences significantly better channel conditions than the other. Under this condition, we have derived a simple rate selection algorithm that improves the fairness with respect to FDM schemes that allocate the same bandwidth to each beam. Leveraging on RS, NCRS is able to provide higher theoretical rates than SC-SCD, while achieving the same rates in degraded channels.

The rest of the paper is organized as follows. Section II introduces the system model. Section III is devoted to computing the rate region of SC-SCD and NCRS. Section IV describes a rate selection algorithm for degraded channels. Finally, Section V provides the numerical results and Section VI derives conclusions.

\section{SYSTEM MODEL}

We consider a multibeam satellite system with $K_{b}$ beams and $K_{b}$ users. In the following we provide the baseband 


$$
\begin{gathered}
\mathcal{R}_{1}\left(\lambda_{1}, \lambda_{2}\right)=\left\{\left(R_{1}, R_{2}\right) \in \mathbb{R}_{+}^{2}: R_{1} \leq \min \left(\log _{2}\left(1+\frac{\lambda_{1} \gamma_{21}+\lambda_{2} \gamma_{22}}{\bar{\lambda}_{1} \gamma_{21}+\bar{\lambda}_{2 \gamma_{22}+1}}\right), \log _{2}\left(1+\frac{\lambda_{1} \gamma_{11}+\lambda_{2} \gamma_{12}}{\bar{\lambda}_{1} \gamma_{11}+\bar{\lambda}_{2} \gamma_{12}+1}\right)\right)\right. \\
\left.R_{2} \leq \log _{2}\left(1+\bar{\lambda}_{1} \gamma_{21}+\bar{\lambda}_{2} \gamma_{22}\right)\right\} \\
\mathcal{R}_{2}\left(\lambda_{1}, \lambda_{2}\right)=\left\{\left(R_{1}, R_{2}\right) \in \mathbb{R}_{+}^{2}: R_{1} \leq \log _{2}\left(1+\lambda_{1} \gamma_{11}+\lambda_{2} \gamma_{12}\right)\right. \\
\left.R_{2} \leq \min \left(\log _{2}\left(1+\frac{\bar{\lambda}_{1} \gamma_{11}+\bar{\lambda}_{2} \gamma_{12}}{\lambda_{1} \gamma_{11}+\lambda_{2} \gamma_{12}+1}\right), \log _{2}\left(1+\frac{\bar{\lambda}_{1} \gamma_{21}+\bar{\lambda}_{2} \gamma_{22}}{\lambda_{1} \gamma_{21}+\lambda_{2} \gamma_{22}+1}\right)\right)\right\} .
\end{gathered}
$$

model that describes the input-output relation in the discretetime domain, assuming perfect synchronization. Since all beams are generated from a central gateway, the signal timing and frequency alignment can be achieved. The differential timing delay and frequency uncertainty that is introduced by satellite could be a source of impairment that would require compensation. In other words, receiver is assumed perfectly synchronized (in time and frequency) to the received signals, which in their turn are assumed to be perfectly aligned (in time and frequency). Building upon this premise, symbol-rate samples at the matched filter output are expressed as

$$
y_{q}[k]=h_{q q} x_{q}[k]+\sum_{m \in \mathbb{A}_{q}} h_{q m} x_{m}[k]+\eta_{q}[k],
$$

for $q=1, \ldots, K_{b}$. Let $x_{m}[k]$ denote the sequence of symbols that is fed into the $m$-th antenna beam. For the ease of exposition, we assume that users within the same beam are served in a time division multiplexing fashion. In notation terms, let $h_{q m}$ be the channel between the $m$-th antenna beam and the user terminal (UT) located in the spot-beam area illuminated by the $q$-th antenna beam. The set $\mathbb{A}_{q}$ gathers the indexes of the co-channel beams that distort the signal received by the $q$-th UT. The term $\eta_{q}[k]$ represents the variance- $\sigma_{q}^{2}$ additive noise that affects user $q$. The channel coefficient $h_{q m}$, which results from the radiation pattern and the path loss, can be expressed as [1]:

$$
h_{q m}=\mu_{q} e^{j \phi_{q m}} \frac{\sqrt{G_{R} G_{q m}}}{4 \pi d_{q} / \lambda} .
$$

The term $G_{R}$ stands for the receive antenna gain and $G_{q m}$ represents the satellite antenna gain from beam $m$ to the $q$-th UT. As for the rest of the terms, $\lambda$ is the carrier wavelength and $\phi_{q m}$ is the payload phase offset between the $m$-th beam and the $q$-th beam. Finally, the tuple $\left(\mu_{q}, d_{q}\right)$ denotes the atmospheric fading that affects the $q$-th user and the distance from the satellite to the $q$-th user.

For simplicity, we will restrict the analysis to the case $K_{b}=$ 2. The input-output relation of the two-beam system with two receivers is given by (time indexes omitted)

$$
\begin{aligned}
& y_{1}=h_{11} x_{1}+h_{12} x_{2}+\eta_{1} \\
& y_{2}=h_{21} x_{1}+h_{22} x_{2}+\eta_{2} .
\end{aligned}
$$

In the satellite communication system under study, beams are allowed to cooperate but the power allocated to each beam is fixed. Hence, the transmit covariance matrix $\boldsymbol{Q}=\mathbb{E}\left[\boldsymbol{x} \boldsymbol{x}^{\mathrm{H}}\right]$ has to satisfy

$$
\boldsymbol{Q}=\left[\begin{array}{cc}
P_{1} & \nu \\
\nu^{*} & P_{2}
\end{array}\right] \quad\left(|\nu| \leq \sqrt{P_{1} P_{2}}\right),
$$

where $\boldsymbol{x}=\left[\begin{array}{ll}x_{1} & x_{2}\end{array}\right]^{\top}$. Furthermore, we define the signal-tonoise ratio (SNR) of each link as

$$
\gamma_{q m}=\frac{P_{m}\left|h_{q m}\right|^{2}}{\sigma_{q}^{2}}, \quad(q, m \in\{1,2\}) .
$$

\section{CODING SCHEMES}

This section is devoted to computing the rate region achieved by SC-SCD and NCRS in the two-beam system described by (3) and (4). The complexity of these schemes is reasonable, because the number of beams that cooperate is limited to two.

For the sake of the analytical tractability, we limit the analysis to the Gaussian setting. We assume that the channel varies on a time scale much larger than the codeword length (quasi-static fading) and the transmitter is assumed to be cognizant of the channel gains.

\section{A. $S C-S C D$}

SC-SCD is a suitable scheme for degraded broadcast channels, where one receiver is markedly "stronger" than the other due to better channel conditions. The decoding strategy to harness the asymmetry consists in treating the message of the weak user as public and the message of the strong user as private. It is important to remark that the rate assignment for the public message should be selected accordingly to be decodable by both users, while private message is only decodable by the intended user. In such a setting, the strong user recovers both messages by SCD, while the weak user only recovers the public message treating the private message as noise [5]. In the multibeam satellite context, users cannot be always ordered from the strongest to the weakest in a natural way. However, SC-SCD is an appealing strategy despite being suboptimal, because makes possible the use of encoding schemes that are insensitive to the channel phase.

Under Gaussian signaling, SC can be implemented by splitting the transmit vector into two statistically independent Gaussian signals as $\boldsymbol{x}=\boldsymbol{x}_{1}+\boldsymbol{x}_{2}$, where $\boldsymbol{x}_{1}=\left[\begin{array}{ll}x_{11} & x_{12}\end{array}\right]^{\top}$ is intended to user 1 and $\boldsymbol{x}_{2}=\left[\begin{array}{ll}x_{21} & x_{22}\end{array}\right]^{\top}$ is intended to 
user 2. Symbol vectors are distributed as $\boldsymbol{x}_{m} \sim \mathcal{C N}\left(\mathbf{0}, \boldsymbol{Q}_{m}\right)$. Therefore, the sum of covariance matrices $Q=Q_{1}+Q_{2}$ has to satisfy (5). Interestingly, if we impose a diagonal structure on $Q_{1}$ and $Q_{2}$, the rate region is independent of the phases. To prove this, let us parametrize the covariance matrices as

$$
\boldsymbol{Q}_{1}=\left[\begin{array}{cc}
P_{1} \lambda_{1} & 0 \\
0 & P_{2} \lambda_{2}
\end{array}\right] \quad \boldsymbol{Q}_{2}=\left[\begin{array}{cc}
P_{1} \bar{\lambda}_{1} & 0 \\
0 & P_{2} \bar{\lambda}_{2}
\end{array}\right],
$$

with $0 \leq \lambda_{1}, \lambda_{2} \leq 1$, where $\bar{\lambda}_{1}=1-\lambda_{1}$ and $\bar{\lambda}_{2}=1-\lambda_{2}$. Assuming that user 1 plays the role of the weak user, the rate region achieved by SC-SCD is given by (8). First of all, both receivers jointly decode $x_{11}$ and $x_{12}$ treating the interference as noise. Then, user 2 applies SCD and jointly detects $x_{21}$ and $x_{22}$ without interference. Exchanging the roles of users 1 and 2 , the rate region is given by (9). The overall rate region is the convex closure of the union of regions (8) and (9), namely,

$$
\mathcal{R}=\operatorname{conv}\left\{\bigcup_{0 \leq \lambda_{1}, \lambda_{2} \leq 1} \mathcal{R}_{1}\left(\lambda_{1}, \lambda_{2}\right) \cup \mathcal{R}_{2}\left(\lambda_{1}, \lambda_{2}\right)\right\} .
$$

From here onwards we assume that the broadcast channel is degraded, so that user 2 experiences better channel conditions, i.e., $\min \left(\gamma_{21}, \gamma_{22}\right) \geq \max \left(\gamma_{11}, \gamma_{12}\right)$. In addition, we will take for granted that the signal received from the reference beam has higher SNR than that received from the adjacent beam. Hence,

$$
\gamma_{12} \leq \gamma_{11} \leq \gamma_{21} \leq \gamma_{22} .
$$

Proposition 1. If (11) holds, then

$$
\mathcal{R}=\operatorname{conv}\left\{\bigcup_{0 \leq \lambda_{1}, \lambda_{2} \leq 1} \mathcal{R}_{1}\left(\lambda_{1}, \lambda_{2}\right)\right\} .
$$

Proof: Let us define

$$
\begin{gathered}
R_{a}\left(\lambda_{1}, \lambda_{2}\right)=\log _{2}\left(1+\frac{\lambda_{1} \gamma_{21}+\lambda_{2} \gamma_{22}}{\bar{\lambda}_{1} \gamma_{21}+\bar{\lambda}_{2} \gamma_{22}+1}\right) \\
R_{b}\left(\lambda_{1}, \lambda_{2}\right)=\log _{2}\left(1+\frac{\lambda_{1} \gamma_{11}+\lambda_{2} \gamma_{12}}{\bar{\lambda}_{1} \gamma_{11}+\bar{\lambda}_{2} \gamma_{12}+1}\right) \\
R_{c}\left(\lambda_{1}, \lambda_{2}\right)=\log _{2}\left(1+\bar{\lambda}_{1} \gamma_{21}+\bar{\lambda}_{2} \gamma_{22}\right) .
\end{gathered}
$$

It must be highlighted that $R_{a}\left(\lambda_{1}, \lambda_{2}\right)=R_{b}\left(\lambda_{1}, \lambda_{2}\right)$, for $\lambda_{2}=$ $\alpha \lambda_{1}$, where

$$
\alpha=\frac{1+\frac{\gamma_{11}-\gamma_{21}}{\gamma_{11} \gamma_{22}-\gamma_{12} \gamma_{21}}}{1+\frac{\gamma_{22}-\gamma_{12}}{\gamma_{11} \gamma_{22}-\gamma_{12} \gamma_{21}}} .
$$

From (11) and (16), we can infer that $\alpha \leq 1$. Then, under the condition (11), two cases can be differentiated in (8), namely,

1) $\lambda_{2} \leq \alpha \lambda_{1}$ and $0 \leq \lambda_{1}, \lambda_{2} \leq 1$

In this case, $\min \left(R_{a}\left(\lambda_{1}, \lambda_{2}\right), R_{b}\left(\lambda_{1}, \lambda_{2}\right)\right)=R_{a}\left(\lambda_{1}, \lambda_{2}\right)$ and $\log _{2}\left(1+\gamma_{11}+\gamma_{12}\right) \leq R_{a}\left(\lambda_{1}, \lambda_{2}\right)+R_{c}\left(\lambda_{1}, \lambda_{2}\right)=\log _{2}(1+$ $\left.\gamma_{21}+\gamma_{22}\right)$. Thus, the maximum attainable rates in (8) are given by $R_{1}=R_{a}\left(\lambda_{1}, \lambda_{2}\right)$ and $R_{2}=R_{c}\left(\lambda_{1}, \lambda_{2}\right)$.

2) $\alpha \lambda_{1}<\lambda_{2}$ and $0 \leq \lambda_{1}, \lambda_{2} \leq 1$.

In this case, $\min \left(R_{a}\left(\lambda_{1}, \lambda_{2}\right), R_{b}\left(\lambda_{1}, \lambda_{2}\right)\right)=R_{b}\left(\lambda_{1}, \lambda_{2}\right)$ and $\log _{2}\left(1+\gamma_{11}+\gamma_{12}\right) \leq R_{b}\left(\lambda_{1}, \lambda_{2}\right)+R_{c}\left(\lambda_{1}, \lambda_{2}\right)<\log _{2}(1+$ $\left.\gamma_{21}+\gamma_{22}\right)$. Thus, the maximum attainable rates in (8) are given by $R_{1}=R_{b}\left(\lambda_{1}, \lambda_{2}\right)$ and $R_{2}=R_{c}\left(\lambda_{1}, \lambda_{2}\right)$.

It has been demonstrated that

$$
\begin{aligned}
\mathcal{R}_{2}^{\mathrm{ub}} & =\left\{\left(R_{1}, R_{2}\right) \in \mathbb{R}_{+}^{2}: R_{1}+R_{2} \leq \log _{2}\left(1+\gamma_{11}+\gamma_{12}\right)\right\} \\
& \subseteq\left\{\bigcup_{0 \leq \lambda_{1}, \lambda_{2} \leq 1} \mathcal{R}_{1}\left(\lambda_{1}, \lambda_{2}\right)\right\}=\mathcal{R}_{1} .
\end{aligned}
$$

In other words, any boundary point of $\mathcal{R}_{1}$ has a sum-rate higher than $\log _{2}\left(1+\gamma_{11}+\gamma_{12}\right)$. In addition, it can be readily verified that

$$
\mathcal{R}_{2}=\left\{\bigcup_{0 \leq \lambda_{1}, \lambda_{2} \leq 1} \mathcal{R}_{2}\left(\lambda_{1}, \lambda_{2}\right)\right\} \subseteq \mathcal{R}_{2}^{\mathrm{ub}} .
$$

From (17) and (18) it follows that $\mathcal{R}_{2} \subseteq \mathcal{R}_{1}=\mathcal{R}$. Therefore, we can state that Proposition 1 holds true.

\section{B. NCRS}

$\mathrm{ENH}$ is presented as a way to increase the GDoF when only the coarse strength of the signals are available at the transmit side [6]. In the 2 user MISO-BC, the rationale behind ENH is to split one selected user's message into a public and a private message. The main idea of ENH is to send the public message from both beams so that it is possible to leverage the interfering signal. Unfortunately, ENH actual performance depends on how both received versions of the public message combine, i.e., it is determined by the channel phase. To avoid the channel dependence, NCRS is presented. Similarly to ENH, one of the user's messages is split into a public and a private message, but then the public message is again split and each of the parts is fed into a different beam. Suppose that RS is applied to user 1. In this case, the public message intended to user 1 is encoded into $x_{1 c}$ and $x_{2 c}$. If $x_{i p}$ denotes the encoded signal that conveys the private message of the $i$-th user, then the transmitted signals read as

$$
\begin{aligned}
& x_{1}=\sqrt{P_{1} \lambda_{1}} x_{1 c}+\sqrt{P_{1} \bar{\lambda}_{1}} x_{1 p} \\
& x_{2}=\sqrt{P_{2} \lambda_{2}} x_{2 c}+\sqrt{P_{2} \bar{\lambda}_{2}} x_{2 p},
\end{aligned}
$$

with $\mathbb{E}\left[\left|x_{i p}\right|^{2}\right]=1$ and $\mathbb{E}\left[\left|x_{i c}\right|^{2}\right]=1$, for $i=1,2$. Since the signals are statistically independent, it follows that $\mathbb{E}\left[\left|x_{1}\right|^{2}\right]=$ $P_{1}, \mathbb{E}\left[\left|x_{2}\right|^{2}\right]=P_{2}$ and $\mathbb{E}\left[x_{1} x_{2}^{*}\right]=\mathbb{E}\left[x_{1}^{*} x_{2}\right]=0$.

The receivers first jointly decode the signals $x_{1 c}$ and $x_{2 c}$ treating the interference as noise. Then, user 1 (user 2) applies SCD and recovers the corresponding private message from $x_{1 p}$ $\left(x_{2 p}\right)$ in the absence of interference.

The NCRS rate region $\mathcal{R}_{1}\left(\lambda_{1}, \lambda_{2}\right)$ and $\mathcal{R}_{2}\left(\lambda_{1}, \lambda_{2}\right)$ for Gaussian codebooks, which are obtained when the public message is assigned to user 1 and to user 2 respectively, are given by (21) and (22). Borrowing the notation from Section III-A, the achievable rate region can formulated by (10). It must be mentioned that (21) and (22) are only valid if $R_{1}$ and $R_{2}$ are different from zero. If $R_{1}=0$ or $R_{2}=0$, then all timefrequency resources are given to one user, which means that the public message has to be only recovered at the intended destination. 


$$
\begin{gathered}
\mathcal{R}_{1}\left(\lambda_{1}, \lambda_{2}\right)=\left\{\left(R_{1}, R_{2}\right) \in \mathbb{R}_{+}^{2}: R_{1} \leq \log _{2}\left(1+\frac{\bar{\lambda}_{1} \gamma_{11}}{1+\bar{\lambda}_{2} \gamma_{12}}\right)+\min \left(\log _{2}\left(1+\frac{\lambda_{1} \gamma_{11}+\lambda_{2} \gamma_{12}}{1+\bar{\lambda}_{1} \gamma_{11}+\bar{\lambda}_{2} \gamma_{12}}\right), \log _{2}\left(1+\frac{\lambda_{1} \gamma_{21}+\lambda_{2} \gamma_{22}}{1+\bar{\lambda}_{1 \gamma_{21}}+\bar{\lambda}_{2} \gamma_{22}}\right)\right)\right. \\
\left.R_{2} \leq \log _{2}\left(1+\frac{\bar{\lambda}_{2} \gamma_{22}}{1+\bar{\lambda}_{1} \gamma_{21}}\right)\right\} \\
\mathcal{R}_{2}\left(\lambda_{1}, \lambda_{2}\right)=\left\{\left(R_{1}, R_{2}\right) \in \mathbb{R}_{+}^{2}: R_{1} \leq \log _{2}\left(1+\frac{\bar{\lambda}_{1} \gamma_{11}}{1+\bar{\lambda}_{2} \gamma_{12}}\right)\right. \\
\left.R_{2} \leq \log _{2}\left(1+\frac{\bar{\lambda}_{2} \gamma_{22}}{1+\bar{\lambda}_{1} \gamma_{21}}\right)+\min \left(\log _{2}\left(1+\frac{\lambda_{1} \gamma_{11}+\lambda_{2} \gamma_{12}}{1+\bar{\lambda}_{1} \gamma_{11}+\bar{\lambda}_{2} \gamma_{12}}\right), \log _{2}\left(1+\frac{\lambda_{1} \gamma_{21}+\lambda_{2} \gamma_{22}}{1+\bar{\lambda}_{1} \gamma_{21}+\bar{\lambda}_{2} \gamma_{22}}\right)\right)\right\}
\end{gathered}
$$

\section{RATE SELECTION FOR DEGRADED CHANNELS}

Among all the possible rate pairs that guarantee fairness and avoid trivial rate assignments, we shall select the one that maximizes the sum-rate. At first sight, we can resolve that this is not a trivial task. In this regard, this section highlights some ideas to simplify the rate selection for degraded channels. First of all we tackle the rate selection in SC-SCD and then we extend the results to NCRS.

To compute the rate selection we stick to the degraded channel condition formulated in (11). For this reason, (9) will be neglected when performing the rate selection. Then, a plausible strategy that strikes a good balance between fairness and throughput consists in computing the rates as follows:

$$
\begin{array}{cl}
\underset{0 \leq \lambda_{1}, \lambda_{2} \leq 1}{\operatorname{argmax}} & R_{1}+R_{2} \\
\text { s.t. } & R_{1}=\min \left(R_{b}\left(\lambda_{1}, \lambda_{2}\right), R_{a}\left(\lambda_{1}, \lambda_{2}\right)\right) \\
& R_{2}=R_{c}\left(\lambda_{1}, \lambda_{2}\right)=\frac{1}{2} \log _{2}\left(1+2 \gamma_{22}\right) .
\end{array}
$$

Note that the constraints ensure that the rate assigned to the strong user would coincide with that achieved in FDM schemes, provided that the bandwidth is equally split among beams. In the numerical results section it will be shown that this constraint guarantees that fairness can be improved when compared to FDM. The problem boils down to solving

$$
\begin{array}{cl}
\underset{0 \leq \lambda_{1}, \lambda_{2} \leq 1}{\operatorname{argmax}} & \min \left(R_{b}\left(\lambda_{1}, \lambda_{2}\right), R_{a}\left(\lambda_{1}, \lambda_{2}\right)\right) \\
\text { s.t. } & \lambda_{2}=1-\frac{2^{R_{s}}-1-\bar{\lambda}_{1} \gamma_{21}}{\gamma_{22}} \\
& R_{s}=\frac{1}{2} \log _{2}\left(1+2 \gamma_{22}\right) .
\end{array}
$$

After several derivation steps, it can be verified that

$$
\begin{aligned}
& R_{a}\left(\lambda_{1}, \lambda_{2}\right)=\log _{2}\left(1+\gamma_{21}+\gamma_{22}\right)-R_{s} \\
& R_{b}\left(\lambda_{1}, \lambda_{2}\right)= \\
& \log _{2}\left(1+\frac{\lambda_{1}\left(\gamma_{11} \gamma_{22}-\gamma_{12} \gamma_{21}\right)-\gamma_{12}\left(2^{R_{s}}-1-\gamma_{21}-\gamma_{22}\right)}{\lambda_{1}\left(\gamma_{11} \gamma_{22}-\gamma_{12} \gamma_{21}\right)+\gamma_{22}+\gamma_{12}\left(2^{R_{s}}-1\right)}\right),
\end{aligned}
$$

when $\lambda_{2}=1-\frac{2^{R_{s}}-1-\bar{\lambda}_{1} \gamma_{21}}{\gamma_{22}}$. As a consequence, the objective function of the maximization problem posed in (24) is monotonically increasing with $\lambda_{1}$. Hence, if $\lambda_{1}=1$ belongs to the feasible set, then the optimal solution is given by

$$
\begin{aligned}
R_{2}= & \frac{1}{2} \log _{2}\left(1+2 \gamma_{22}\right) \\
R_{1}= & \min \left(\log _{2}\left(1+\frac{\gamma_{11} \gamma_{22}-\gamma_{12}\left(2^{R_{2}}-1-\gamma_{22}\right)}{\gamma_{22}+\gamma_{12}\left(2^{R_{2}}-1\right)}\right)\right. \\
& \left., \log _{2}\left(1+\gamma_{21}+\gamma_{22}\right)-R_{2}\right) .
\end{aligned}
$$

Interestingly, $\lambda_{1}=1$ belongs to the feasible set if $\frac{1}{2} \log _{2}(1+$ $\left.2 \gamma_{22}\right) \leq \log _{2}\left(1+\gamma_{22}\right)$. We can resolve that the inequality will be always satisfied because the first derivative of $\log _{2}\left(1+\gamma_{22}\right)-\frac{1}{2} \log _{2}\left(1+2 \gamma_{22}\right)$ is monotonically increasing, for $\gamma_{22}>0$. Accordingly, (26) will dictate the rate selection.

It is noteworthy to mentioning that if we set $\lambda_{1}=1$, then (8) and (21) are equivalent. Hence, we can state that rate pairs satisfying (26) are achievable by NCRS as well.

\section{NUMERICAL RESULTS}

This section provides some numerical results for the twouser communication system described in Section II. Although this is a simplified scenario, it provides valuable insights regarding in comparative assessment of different access scheme strategies. Concerning the channel model, it has been assumed that the envelope of the channel is a nonrandom constant and thus, the impact of the fading has not been evaluated. In this regard, the scenario can be parametrized by considering the ratios defined in (6). The most representative schemes to compare SC-SCD and NCRS with are described in the following.

Frequency division multiplexing (FDM). Two users are simultaneously served in non-overlapping frequency bands. The rate region is given by

$$
R_{1}<\lambda \log _{2}\left(1+\frac{\gamma_{11}}{\lambda}\right), \quad R_{2}<\bar{\lambda} \log _{2}\left(1+\frac{\gamma_{22}}{\bar{\lambda}}\right),
$$

for $0 \leq \lambda \leq 1$ and $\bar{\lambda}=1-\lambda$.

Time-sharing (TS). Both transmitters cooperate to serve the first user for a fraction $\lambda$ of the total time, and do the same with the second user for the remaining $1-\lambda$ fraction of the time [7], [8]. The rates can be formulated as

$$
R_{1} \leq \lambda \log _{2}\left(1+\gamma_{11}+\gamma_{12}\right), \quad R_{2} \leq \bar{\lambda} \log _{2}\left(1+\gamma_{21}+\gamma_{22}\right),
$$

for $0 \leq \lambda \leq 1$ and $\bar{\lambda}=1-\lambda$.

Han-Kobayashi (HK). We consider a special case among the schemes introduced by Han and Kobayashi [9]. It is important to remark that the interference channel is used as reference and thus, beams are not allowed to cooperate. The strategy that is considered involves splitting the information into one public part and another private that are sent via SC. The idea is that the public information is decoded by both receivers while the private information is decoded only at the intended receiver. The rate region is contained within the set 


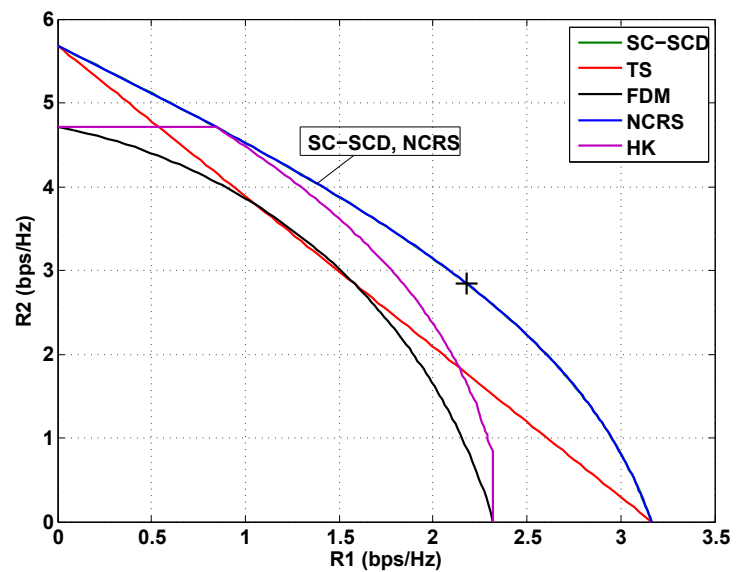

Figure 1. Rate region for $\gamma_{11}=\gamma_{12}=6 \mathrm{~dB}, \gamma_{22}=\gamma_{21}=14 \mathrm{~dB}$.

of rate pairs satisfying [5, Theorem 6.4]. As compared to the general scheme, we have dropped the time-sharing variable.

The first test was performed for an asymmetric configuration where (11) is satisfied. The scenario simulated in Figure 1 corresponds to the case where both users are suffering from high interference and the SNRs are unbalanced. As Figure 1 shows, the rate region achieved by SC-SCD and NCRS coincide. We have represented with a marker the rate pair that stems from (26), resulting in $R_{1}=2.18$ and $R_{2}=2.83$ $\mathrm{bps} / \mathrm{Hz}$. By exploiting the interference, $R_{1}$ is increased by $37 \%$ with respect to FDM, which improves the sum-rate and the fairness. The plots also highlight that the proposed schemes clearly outperform other strategies that either do not leverage on RS or do not allow full beam cooperation.

The scenario simulated in Figure 2 corresponds to a symmetric configuration, i.e., both direct links have the same magnitude, and the interfering links are also equal in magnitude. Under these conditions, SC-SCD gives poor performance. The observation could be predicted knowing beforehand that the channel simulated in Figure 2 is not degraded. Interestingly, NCRS benefits from RS to achieve the largest rate region even when the interference vanishes.

It should be noted that in a realistic multibeam communication satellite system, a variety of user pairs with different link and interference conditions can be identified. Taking into account fairness capacity distribution among users, the two illustrative examples presented above both indicate that access strategies such as SC-SCD and NCRS can enhance the sumrate capacity compared to that of orthogonal scheme. However, the level of improvement is highly dependent on the channel matrix and the design of user-pairing strategies, which is left for future work. Accordingly, the analysis is restricted to the two-user case.

\section{CONCLUSIONS}

This paper explores the applicability of SC and RS in a two-beam satellite communication system. Especial emphasis has been given to low-complexity encoding schemes that do not require the channel phase information. The theoretical

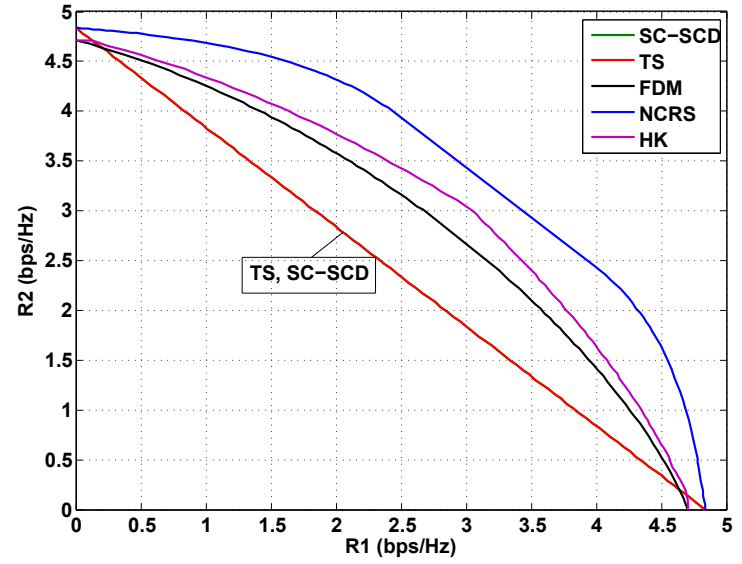

Figure 2. Rate region for $\gamma_{11}=\gamma_{22}=14 \mathrm{~dB}, \gamma_{12}=\gamma_{21}=4 \mathrm{~dB}$.

rates achieved by the proposed schemes are higher than those achieved by orthogonal schemes and other solutions that either do not allow beam cooperation or do not apply RS.

\section{ACKNOWLEDGMENT}

This work has been supported by the European Space Agency funded activity SatNEx IV Contract No.4000113177/15/NL/CLP. The views of the authors of this paper do not reflect the views of ESA. This work has received funding from the Ministerio de Ciencia, Innovación y Universidades under project TERESA- TEC201790093-C3-1-R (AEI/FEDER,UE). This work was partially funded by the Agencia Estatal de Investigacion (Spain) and the European Regional Development Fund (ERDF) through the project MYRADA (TEC2016-75103-C2-2-R). Funded also by the Catalan Government under project 2017-SGR-1479.

\section{REFERENCES}

[1] B. Devillers, A. Perez-Neira, and C. Mosquera, "Joint Linear Precoding and Beamforming for the Forward Link of Multi-Beam Broadband Satellite Systems," in IEEE Global Telecommunications Conference (GLOBECOM), Dec. 2011.

[2] G. Taricco, "Linear Precoding Methods for Multi-Beam Broadband Satellite Systems," in European Wireless 2014; 20th European Wireless Conference, May 2014.

[3] M. A. Vazquez, A. Perez-Neira, D. Christopoulos, S. Chatzinotas, B. Ottersten, P. D. Arapoglou, A. Ginesi, and G. Taricco, "Precoding in Multibeam Satellite Communications: Present and Future Challenges," IEEE Wireless Communications, vol. 23, no. 6, pp. 88-95, Dec. 2016.

[4] B. Clerckx, H. Joudeh, C. Hao, M. Dai, and B. Rassouli, "Rate splitting for MIMO wireless networks: a promising PHY-layer strategy for LTE evolution," IEEE Communications Magazine, vol. 54, no. 5, pp. 98-105, May 2016.

[5] A. E. Gamal and Y.-H. Kim, Network Information Theory. New York, NY, USA: Cambridge University Press, 2012.

[6] A. G. Davoodi and S. A. Jafar, "Transmitter Cooperation Under Finite Precision CSIT: A GDoF Perspective," IEEE Transactions on Information Theory, vol. 63, no. 9, pp. 6020-6030, Sept. 2017.

[7] M. Caus, A. I. Perez-Neira, M. Angelone, and A. Ginesi, "An innovative interference mitigation approach for high throughput satellite systems," in 2015 IEEE 16th International Workshop on Signal Processing Advances in Wireless Communications (SPAWC), June 2015.

[8] G. Colavolpe, A. Modenini, A. Piemontese, and A. Ugolini, "Multiuser Detection in Multibeam Satellite Systems: Theoretical Analysis and Practical Schemes," IEEE Trans. Commun., vol. 65, no. 2, pp. 945-955, Feb. 2017.

[9] T. Han and K. Kobayashi, "A new achievable rate region for the interference channel," IEEE Transactions on Information Theory, vol. 27, no. 1, pp. 49-60, Jan. 1981. 